Journal of Computer Science 7 (6): 949-953, 2011

ISSN 1549-3636

(C) 2011 Science Publications

\title{
Highway Traffic Incident Detection using High-Resolution Aerial Remote Sensing Imagery
}

\author{
Seyed Mostafa Mousavi Kahaki and Md. Jan Nordin \\ Department of Artificial Intelligence Technology, Faculty of Information Science and Technology, \\ University Kebangsaan Malaysia, 43600, Bangi, Selangor, Malaysia
}

\begin{abstract}
Problem statement: As vehicle population increases, Intelligent Transportation Systems (ITS) become more significant and mandatory in today's overpopulated world. Vital problems in transportation such as mobility and safety of transportation are considered more, especially in metropolitans and highways. The main road traffic monitoring aims are: the acquisition and analysis of traffic figures, such as number of vehicles, incident detection and automatic driver warning systems are developed mainly for localization and safety purposes. Approach: The objective of this investigation was to propose a strategy for road extraction and incident detection using aerial images. Real time extraction and localization of roadways in an satellite image is an emerging research field which can applied to vision-based traffic controlling and unmanned air vehicles navigation. Results: The results of the proposed incident detection algorithm show that it has good detection performance, the maximum angle of vehicles applied for incidet detection is $45^{\circ}$ and the performance for learning system in order to vehicle detection is $86 \%$. This performance achived in testing algorithm on 45 highway aerial images. Conclusion: In order to consider with the high complexity of this kind of imagery, we integrate knowledge about roadways using formulated scale-dependent models. The intensity images are used for the extraction of road from satellite images. Threshold techniques, neural network and Radon transform are used for the road extraction, vehicle detection and incident detection. Results indicated that in most aerial images the incident can be detect by applying the angle algorithm.
\end{abstract}

Key words: Incident detection, vehicle detection, remote sensing imagery, traffic monitoring, highresolution aerial, radon transform, detection algorithm

\section{INTRODUCTION}

Traffic controlling and incident detection is an emerging research topic which is rapidly increasing interest in traffic controlling systems. Currently, control of traffic is a difficult and time consuming task that need too several human operators. Real time traffic controlling using satellite images can save time and costs. The urban traffic control process is accomplished through cameras which are installed in highways, at the moment. Recently, with considering high-resolution aerial imageries, existence of an intelligence road extraction and vehicle detection system which is be able to control the road traffic has had more remarkable performance. Investigations about road extraction and vehicle detection in aerial imageries involved in information and data related to GIS and this maintained data needs to become up to date in every certain period of time.

Road extraction and vehicle detection for incident detection in aerial imageries is a newly controversial issue in computer vision that has also some influences many other projects and operations such as traffic controlling and incident detection in highways. Some realtime applications using ground camera has been implemented for traffic such as (AlQahtani, 2006; Hirzallah, 2007; Panich, 2010; Zargari et al., 2009). Since the developement of new satellite systems such as Quick Bird, IKONOS and Geoeye-1, the remote sensing imagery are available with 0.25 meter resolution. Vehicles can observed clearly on these kind of satellite imagery. The results of proposed algorithm show that it has good detection performance. Some vehicle detection methods have been studied using aerial imagery (Zheng and Li , 2007; Zhenfeng et al., 2004). The major factors that affect essentially on our subject are: the number of objects which exist in the input image, the total number of relationship between them and also some properties that distinguish them from other objects. Example aerial image for incident detection are shown in Fig. 1.

Corresponding Author: Seyed Mostafa Mousavi Kahaki, Department of Artificial Intelligence Technology,

Faculty of Information Science and Technology, University Kebangsaan Malaysia, 43600, Bangi, Selangor, Malaysia 


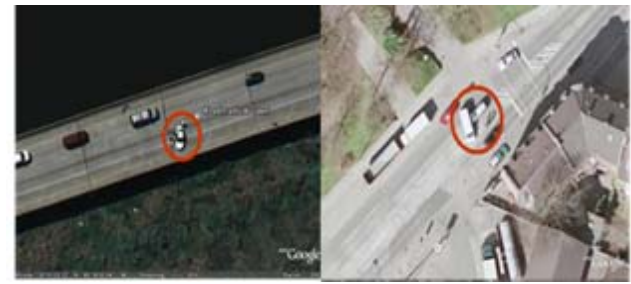

Fig. 1: An example of aerial image for incident detection

Before explaining any details, we scan other parts of this article:

Sec. Material and methods: Important Issues in road Extraction and vehicle detection process. Main idea, road and vehicle detection for incident detection, essential items and their characteristics and execution on aerial imageries,

Sec. Results: The experiment results of proposed algorithm.

Sec. Discussion: The performance of the system and advantages and disadvantages of system.

Sec. Conclusion: A proposal about works for future on this topic

\section{MATERIALS AND METHODS}

Vehicle detection and road extraction have been receiving attention in the computer vision. A number of conventional high way incident detection algorithms have been developed in the past several years. Techniques based on morphology and neural network for vehicle detection and road extraction had developed in machine vision, but only a few researchers have investigated the detection of traffic sensing and incident detection on aerial images (Lairong et al., 2010). In this study we used high resolution images from Geoeye-1 satellite. This imagery are taken full color in equal interval from the ground. GeoEye-1 is equipped with the newest technology ever used in satellite systems. It offers resolution by acquiring 0.25 -meter panchromatic and 1.65-meter multispectral imagery. The used method is presented in Fig. 2 as a flowchart.

First, we must extract the roads and distinguish coordinates of road's district and then the vehicle detection's operation is accomplished. In addition, we need feature extraction of road because after detection of road's district, only those objects can be studied that are inside the road district. This is really useful to know that roads are presents as a straight district with different color in the satellite imagery. Therefore, linear feature can be an appropriate feature in detection at road's district. Another feature which roads possess is the lines that exist in white color in all the roads and they are considered as a proper feature in detection operation.

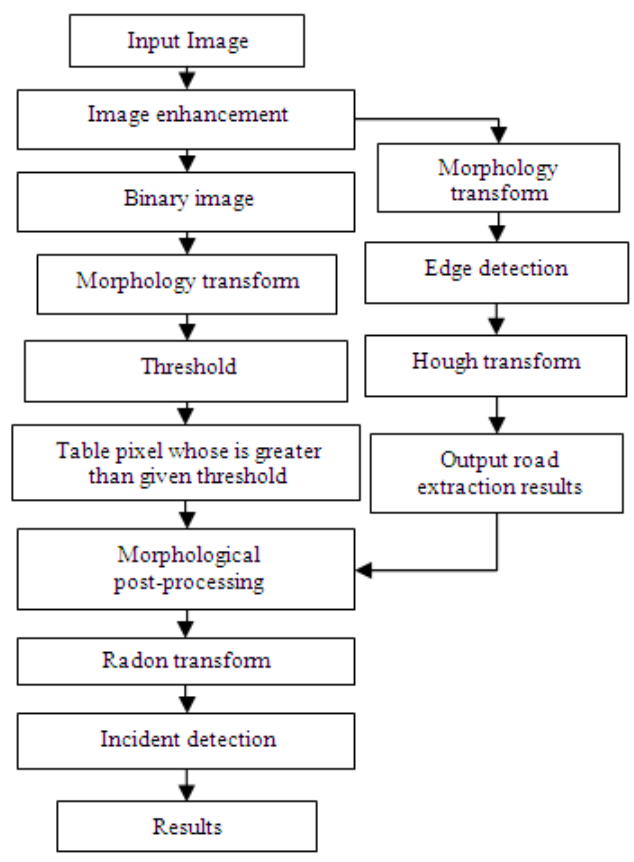

Fig. 2: Flowchart of the incident detection algorithm

These lines are available continuously alongside the road and discontinuously in the middle at the road. The other feature used in this method is the color of the road which distinguishes road from the edge completely specified. With threshold operation, we can separate road and background from each other and preparing images for other operations. This color threshold can be approached with mean of colors at the roads in several aerial imageries that are available in dataset. After threshold, edge detection operation can be run better. We had used the canny method for edge detection. This method has higher performance rather than the others. In digital imageries, in those points that there is edge on them, there are differences of color too. Therefore, sharpen operation with a filter leads to increase the difference at color in edges and it can enhance the image classification. As result, next operation like edge detection can be executed with higher precision. The equations for edge detection are shown on Eq. 1-5:

$$
\begin{aligned}
& \frac{d f(x)}{d x}=\lim _{\Delta x \rightarrow 0} \frac{f(x+\Delta x)-f(x)}{\Delta x} \\
& \frac{d f(x)}{d x} \cong \frac{f(x+1)-f(x-1)}{2}
\end{aligned}
$$

$\mathrm{Gx}=(\mathrm{Z7}+2 \mathrm{Z8}+\mathrm{Z9})-(\mathrm{Z1}+2 \mathrm{Z2}+\mathrm{Z3})$

$\mathrm{Gy}=(\mathrm{Z3}+2 \mathrm{Z6}+\mathrm{Z9})-(\mathrm{Z1}+2 \mathrm{Z4}+\mathrm{Z7})$ 


$$
\Delta \mathrm{f} \approx|\mathrm{Gx}|+|\mathrm{Gy}|
$$

The Eq. 1-2 are mathematic edge detection and 3, 4 and 5 are the filters to implement edge detection on images.

In the next step, we use Hough transform method to distinguished road in aerial images.

After road extraction, we need to detect the vehicles in the road. This trend is moves simply because only those objects which are inside the roads can be handled. One of the important textures of vehicles, which helps us to detect, is vehicle model. We can detect a vehicle with using its model from dataset examples by using neural network classification.

Vehicles should be extracted for incident detection. Vehicles always in aerial imageries are appearing in rectangular shape as you can see in Fig. 3. Then morphology transform is use in order to pre-processing for enhance features of vehicles. It is defined by Gradient Eqn. 6-8:

$G(f)=(f \oplus g)-(f \Theta g)$

Where:

$\mathrm{f} \quad=$ Gray scale input image

$\mathrm{g} \quad=$ A structural element

$\mathrm{f} \oplus \mathrm{g}=$ Represent dilation operation

$\mathrm{f} \Theta \mathrm{g}=$ Means erosion operation (Gonzalez and Woods, 2008)

Dilation:

$$
\mathrm{A} \oplus \mathrm{B}=\left\{\mathrm{z}:(\hat{\mathrm{B}})_{\mathrm{z}} \cap \mathrm{A} \neq \varphi\right\}
$$

Erosion:

The erosion of A by B, written AӨB, is given by :

$$
\mathrm{A} \Theta \mathrm{B}=\left\{\mathrm{z} \mid(\mathrm{B})_{\mathrm{z}} \subseteq \mathrm{A}\right\}
$$

In Fig. 3 shows a Morphology image of TehranKaraj (IRAN) highway. We can use this process in order to discriminate vehicle and non-vehicle points. Fig. 4 shows some dataset examples for neural network classification. One noticeable feature in aerial imageries is the color of the vehicles that has a significant difference with other objects. This phenomenon can helps us to detect the vehicles by adaptive threshold processing and the results of threshold simulation indicate that this method has high performance. After classification with neural network method the direction of vehicles should be finding for incident detection. The result of mean square error for learning system shown in Fig. 5.

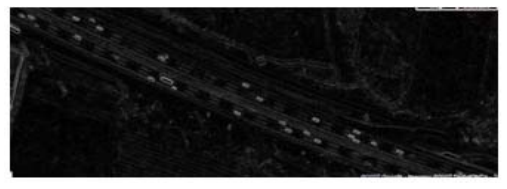

Fig. 3: Morphology preprocessing result

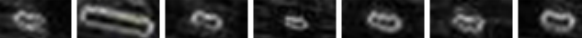

Fig. 4: Dataset examples

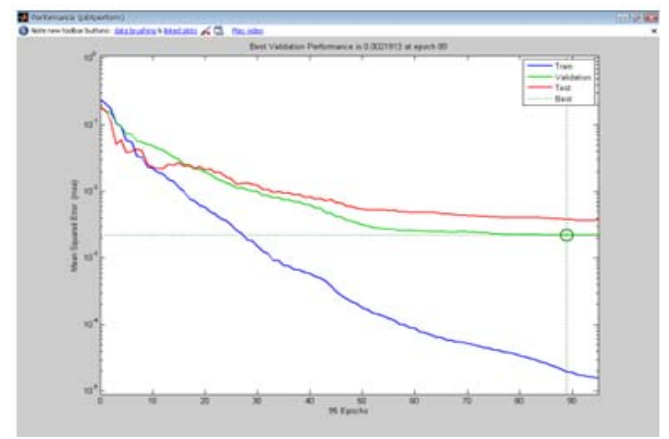

Fig. 5: Mean square error for learning system

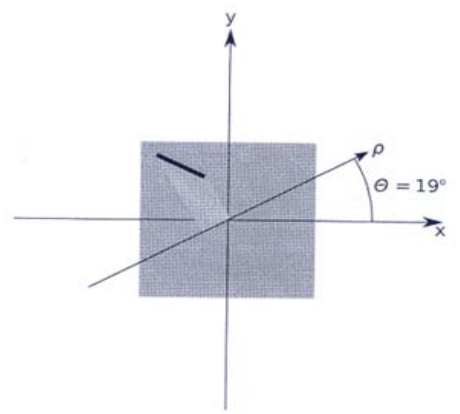

Fig. 6: For example, the line appear in this image has $\Theta=19$

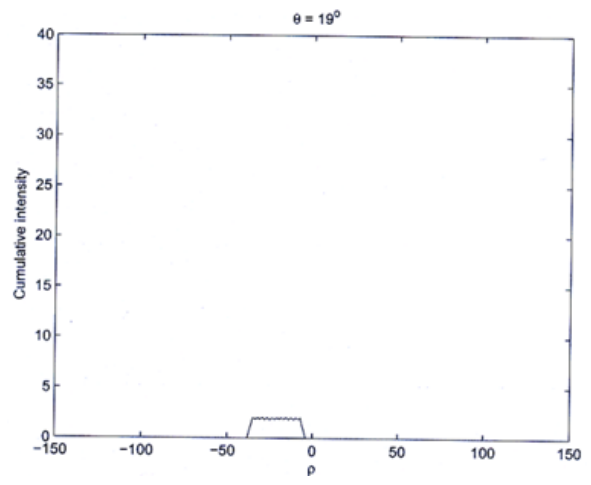

Fig. 7: The result for RT with $\Theta=19$, you can see there is no peak in this result 
The best feature for incident detection is finding directions of vehicles, because the incidents usually happen when there are one or more vehicles with irregular direction in an aerial image of highway. This feature extracted from some aerial images that incident happened on them, so next step after vehicle detection is finding the direction of vehicles. For this goal the skeleton algorithm has been used to transform extracted vehicle to a line and then Radon transform has been used to find the direction of vehicles. In the last step, thresholding is used to find incident, those lines (vehicles) that are in irregular angel can be marked as incident. For this purpose we need to have a mathematical algorithm which can convert lines in original image into special points in the new transformed images. These points need to be local maxima for extract the features with some post-processing. Radon Transform (RT), is a good candidate for this goal. RT can transform the original image into a new image space contain of $\theta$ and $t$ parameters. All the points in the new image represent all information equal to a line in the original image which has angle $\theta$ and radius $t$, this is the best transform we are looking for Fig. 6 and 7 .

By applying the RT on the image $f(x, y)$ for a set of angles can be expect of as computing the projection of the image intensity along a radial line oriented at a specific angle (Metkar and Talbar, 2010). The result of projection is the sum of the intensities of the pixels in directions, i.e. for a line integral (Brady, 1998). The result is a new image $R(\rho, \theta)$. This can be written mathematically by defining Eqn. 9-11:

$\rho=x \cos \theta+y \sin \theta$

Which the RT can be deffined as:

$R(\rho, \theta)=\int_{-\infty}^{\infty} \int_{-\infty}^{\infty} f(x, y) \delta(\rho-x \cos \theta-y \sin \theta) d x d y$

where, $\delta(0)$ is the Dirac delta function.

Radon transform has many properties for varity kind of transform algorithms. RT can find the parameters for any translated, scaled or rotated image by knowing the RT parameters of the original image (Terrades and Valveny, 2003). Rotation property:

$\mathrm{R}\left(\mathrm{f} \circ \mathrm{g}_{\alpha}(\mathrm{x})\right)(\mathrm{t}, \theta)=\mathrm{Rf}(\mathrm{t}, \theta+\alpha)$

The rotation effect on RT can be seen in Fig. 8.a and 8.b., in this image we can see how, when an image rotated, local maxima of RT move to the right. We compare the maximum rotation of lines for incident detection.

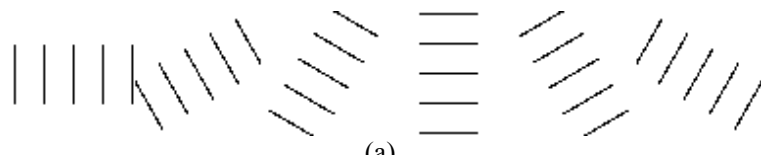

(a)

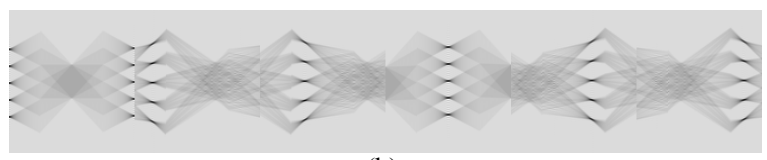

(b)

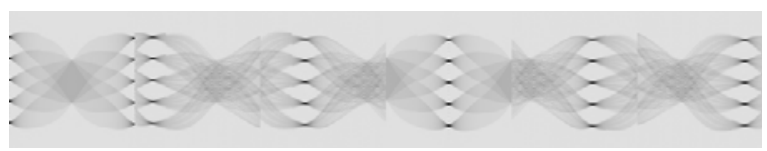

(c)

Fig. 8: (a) The rotated image. (b) The local maximums. (c) The corrected version (Terrades and Valveny, 2003)

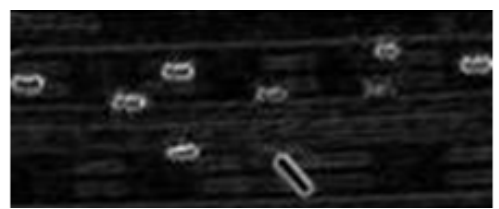

(a)

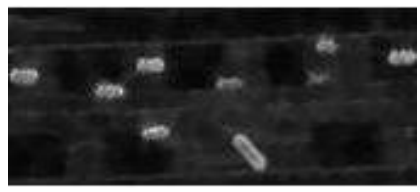

(b)

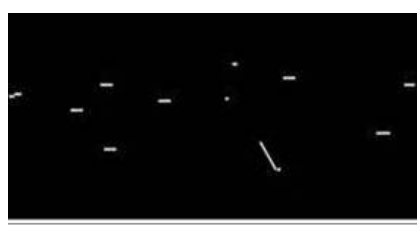

(c)

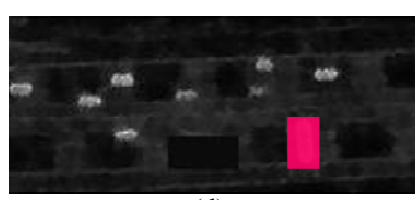

(d)

Fig. 9: Vehicle detection and incident detection result. (a)(b) The sample image of road segments. (c) The vehicle detection result. (d) Incident detection result

\section{RESULTS}

Experiment: by using the proposed incident detection algorithm the incident can be detect in a remote sensing image as shown in Fig. 9. 
Table 1: Incident detection results

\begin{tabular}{lccl}
\hline Site & $\begin{array}{l}\text { Number of } \\
\text { vehicles }\end{array}$ & $\begin{array}{l}\text { Number of } \\
\text { detected vehicles }\end{array}$ & $\begin{array}{l}\text { Incident detection } \\
\text { result (True/False) }\end{array}$ \\
\hline Road1 & 8 & 7 & $\mathrm{~T}$ \\
Road2 & 52 & 47 & $\mathrm{~F}$ \\
Road3 & 31 & 24 & $\mathrm{~T}$ \\
Road4 & 30 & 27 & $\mathrm{~T}$ \\
Road5 & 19 & 17 & $\mathrm{~T}$ \\
Road6 & 55 & 44 & $\mathrm{~F}$ \\
Road7 & 23 & 23 & $\mathrm{~T}$ \\
Road8 & 10 & 10 & $\mathrm{~T}$ \\
Road 9 & 7 & 7 & $\mathrm{~T}$ \\
Road 10 & 14 & 13 & $\mathrm{~T}$ \\
\hline
\end{tabular}

In order to test the system and find the results the images of 10 roadways has been studied and the results of vehicle detection and incident detection on them has shown on Table 1. The result of incident detection is logical values show that the incident detected truly or not.

\section{DISCUSSION}

The results shown that the alghorithm can detect the incident in most of the roadway images. this scheme, of course, has some disadvantages: its performance is $80 \%$ and it cannot detect the vehicles outside the road. This scheme can be accelerated to transfer the information to drivers who intend to cross a roadway and also it can help to the police for traffic controlling. The result of the incident detection is shown in Table 1.

\section{CONCLUSION}

In this study, we focus on the vehicle detection, road extraction and incident detection using high resolution remote sensing imagery for traffic analysis. Further work could include more samples for neural network classification and using more information for shape analysis i.e., edge shapes in order to improve the correct detection rate. This method can use another features for incident detection from high resolution satellite images. Imageries are taken by xerographic satellites and special planes at the moment. We can control the road's traffic via a traffic balloon. Traffic balloons have more flexibility to apply; there is possibility to pursue the roads via traffic balloon as autopilot. Also, we can have several balloons in the roads districts that it is an independent issue. There is need to mention that, this kind of balloons has been already schematized to prepare the aerial imageries (Saiki et al., 2010). Another feature of our system is its capability to following the deviant vehicles which have illegal speed.

\section{REFERENCES}

AlQahtani, S.A., 2006. A simulation-based comparison of multimedia traffic prioritization schemes for high-performance input-queued packet switches. J. Comput. Sci., 2: 347-354. DOI: 10.3844/jcssp.2006.347.354
Brady, M.L., 1998. A fast discrete approximation algorithm for the radon transform. SIAM J. Comput., 27: 107-119. DOI: $10.1137 / \mathrm{S} 0097539793256673$

Gonzalez, R.C. and R.E. Woods, 2008. Digital Image Processing. 3rd Edn., Prentice Hall, USA., ISBN010: 13168728X, pp: 954.

Hirzallah, N., 2007. Automated camera monitoring system for selective areas of interest. J. Comput. Sci., 3: 62-66. DOI: 10.3844/jcssp.2007.62.66

Lairong, C., C. Yuan and J. Ronghua, 2010. Automatic incident detection algorithm based on support vector machine. Proceedings of the 6th International Conference on Natural Computation, Aug. 10-12, IEEE Xplore, Yantai, Shandong, pp: 864-866. DOI: 10.1109/ICNC.2010.5583920

Metkar, S.P. and S.N. Talbar, 2010. Motion detection and projection based block motion estimation using the radon transform for video coding. J. Comput. Sci., 6: 979-986. DOI: 10.3844/jcssp.2010.979.986

Panich, S., 2010. Method of object detection for mobile robot. J. Comput. Sci., 6: 1151-1153. DOI: $10.3844 /$ jcssp.2010.1151.1153

Saiki, H., T. Fukao, T. Urakubo and T. Kohno, 2010. Hovering control of outdoor blimp robots based on path following. Proceedings of the IEEE International Conference on Control Applications, Sept. 8-10, IEEE Xplore, Yokohama, pp: 21242129. DOI: $10.1109 /$ CCA.2010.5611169

Terrades, O.R. and E. Valveny, 2003. Radon transform for lineal symbol representation. Proceedings of the 7th International Conference on Document Analysis and Recognition, Aug. 03-06, Edinburgh, Scotland, pp: 195-195.

Zargari, S.A., M. Araghi and K. Mohammadian, 2009. An application of combined model for tehran metropolitan area incorporating captive travel behavior. Am. J. Applied Sci., 6: 64-71. DOI: 10.3844/ajassp.2009.64.71

Zhenfeng, Z., L. Hanqing, H. James and U. Keiichi, 2004. Car detection based on multi-cues integration. Proceedings of the 17th International Conference on Pattern Recognition, Aug. 23-26, Cambridge UK., pp: 699-702. DOI: 10.1109/ICPR.2004.229

Zheng, H. and L. Li, 2007. Artificial immune approach for vehicle detection from high resolution space imagery. Int. J. Comput. Sci. Network Sec., 7: 67-72. 